\title{
By studying ageing, endocrinology stays young
}

\author{
AJ van der Lely
}

One of the highlights of the latest and very successful annual meeting of the European Society for Endocrinology in Berlin, May 2008, was the charismatic plenary lecture by Nir Barzilai from the Albert Einstein College of Medicine, NY, USA, entitled Why did Moses live to be 120 ? His talk was a perfect example of a hot topic in endocrine research, both in basic and in clinical endocrinology - namely, studying and, if possible, increasing longevity. Indeed, hormones, such as insulin and insulin-like growth factor I (IGF-I), are thought to play major parts in mechanisms related to longevity.

Barzilai and colleagues' work (Rincon $\mathrm{M}$ et al. [2005] Exp Gerontol 40: 873-877), and that of other research groups (e.g. Coschigano KT et al. [2000] Endocrinology 141: 2608-2613), has already addressed several issues and shown clearly that lifespan can be modulated actively by components of the insulin/IGF-I pathway in laboratory animals. Mutations in genes encoding these components result in extension of lifespan in lower species, ranging from yeasts and worms to insects and mice. Human studies, performed at the Albert Einstein College of Medicine, in which sequence analysis was done of the IGF-I and IGF-I-receptor genes in female centenarians, have revealed over-representation of heterozygous mutations in the IGF-I-receptor gene in centenarians compared with in controls. These heterozygous mutations seem to be associated with high levels of IGF-I in serum and reduced activity of the IGF-I receptor, as measured in transformed lymphocytes.

Associations between genetic alterations in the IGF-I receptor and human longevity, such
.... when

moving up the

evolutionary

ladder, genome

complexity

increases and

the impact of

candidate genes

on lifespan

becomes

smaller

AJ van der Lely is an Advisory Board member of Nature Clinical Practice Endocrinology \& Metabolism.

\section{Competing interests}

The author declared no competing interests.

www.nature.com/clinicalpractice doi:10.1038/ncpendmet0942 as those mentioned above, suggest a role for the insulin/IGF-I pathway in modulation of human lifespan. In support of this theory, the key genes that have been identified so far encode proteins with roles in metabolism, maintenance and repair mechanisms that minimize age-related damage becoming permanent through accumulation.

An example of this has been provided by a Danish group, who reported that a well-known functional single-nucleotide polymorphism in the tumor suppressor TP53 gene leads to increased longevity. Among the study participants $(n=9,219)$, selected from the Danish general population, homozygotes for the minor allele versus homozygotes for the major allele had an increase in median survival of 3 years. This group's data indicated that the increased longevity might be due to a generally increased robustness after a diagnosis of any life-threatening disease.

Data have, however, revealed that when moving up the evolutionary ladder, genome complexity increases and the impact of candidate genes on lifespan becomes smaller (Kuningas M et al. [2008] Aging Cell 7: 270-280). Extrapolation of findings from experimental models to humans is further complicated as phenotypes are critically dependent on the setting in which genes are expressed, while laboratory conditions and modern, real-life environments are markedly dissimilar. Endocrinology must, therefore, focus on human models of longevity, and only apply data obtained from animal models with the greatest of care. Studying ageing is tedious work, but it keeps endocrinology young (at heart). 